\title{
Application of Linear Viscoelastic Continuum Damage Theory to the Low and High Strain Rate Response of Thermoplastic Polyurethane
}

\author{
H. Chen ${ }^{1}$ - A.R. Trivedi ${ }^{1}$ - C.R. Siviour ${ }^{1}$ \\ Received: 21 November 2019 / Accepted: 11 May 2020 / Published online: 15 June 2020 \\ (C) The Author(s) 2020
}

\begin{abstract}
Background Understanding the mechanical response of elastomers to applied deformation at different strain rates and temperatures is crucial in industrial design and manufacture; however, this response is often difficult to measure, especially at high strain rates (e.g. $>100 \mathrm{~s}^{-1}$ ), and more predictive methods to obtain constitutive relationships are required.
\end{abstract}

Objective The objective of the research described in this paper is to develop such methods.

Method The paper outlines a novel approach combining quasi-static monotonic tests in tension and compression, quasi-static cyclic tests in tension, and high strain rate tests in compression, with dynamic mechanical analysis and time-temperature superposition. A generalized viscoelastic model incorporating continuum damage is calibrated.

Results The results show that a model calibrated using data from quasi-static compression and dynamic mechanical analysis can be used to adequately predict the compressive high strain rate response: hence, this paper provides an important step in the development of a methodology that avoids the requirement to obtain constitutive data from high strain rate experiments. In addition, data from FE models of the dynamic mechanical analysis experiments are provided, along with a discussion of data obtained from tensile and cyclic loading.

Conclusions The paper demonstrates the effectiveness of 'indirect' predictive methods to obtain information about high rate behaviour of low modulus materials.

Keywords Polyurethane $\cdot$ Dynamic mechanical analysis $\cdot$ High strain-rates · Viscoelasticity $\cdot$ Viscoelastic damage

\section{Introduction}

Thermoplastic elastomers are widely used in engineering and industry, e.g. for bumpers, seals, moulded gaskets and ducts, often working as impact and vibration mitigators. They benefit from advantageous properties such as flexibility, durability and property recovery after deformation, but simul taneously have dependence on polymer network morphology, temperature, strain rate and loading history [1-4].

Electronic supplementary material The online version of this article (https://doi.org/10.1007/s11340-020-00608-2) contains supplementary material, which is available to authorized users.

C.R. Siviour

clive.siviour@eng.ox.ac.uk

1 Department of Engineering Science, University of Oxford, Oxford, OX1 3PJ, UK
The empirical behaviour of many polymers between the rubbery and glassy states has the well-known timetemperature equivalence [5]. In the glassy state, at a low temperature, or during deformation at high rates, the polymer chains have inhibited displacement, which raises the stored elastic energy and stiffens the polymer. In the rubbery state, at elevated temperature, the molecular chains have greater mobility owing to increased free volume and energy, and are able to respond on the loading timescale. This dynamic mechanism also responds at low loading rates. The mechanical interpretation can be extended using the time-temperature superposition (TTS) principle, which describes the equivalence of time and temperature to characterise mechanical properties. Inherent properties, in particular over a range of temperatures near the glass transition, can be mapped onto a master curve which can then be fitted using equations such as the Williams-LandelFerry (WLF) equation (1 below) to build a coordinate 
system for either temperature or frequency dependence of mechanical behaviour [6]. This is done by producing a shift factor, $\log a_{T}$ in equation (1).

$\log a_{T}=-\frac{C_{1}\left(T-T_{r e f}\right)}{C_{2}+\left(T-T_{r e f}\right)}$

where $C_{1}$ and $C_{2}$ are constants, and $T_{r e f}$ is the reference temperature at which the master curve is produced. In this paper, time-temperature equivalence is explored as a means to predict high-rate (e.g. $>700 \mathrm{~s}^{-1}$ ) response of elastomers, using a commercial thermoplastic polyurethane as a model material.

Rate dependence in polyurethanes has been studied using direct measurements over the range of strain rates from $10^{-3} \mathrm{~s}^{-1}$ to $10^{4} \mathrm{~s}^{-1}$ [7-9]. These studies have found that, in common with other polymers, the flow stress magnitude increases with increasing strain rate. Quasi-static experiments may be performed on conventional screwdriven machines, whilst medium and higher strain rates are investigated using hydraulic machines, drop weight instruments, split-Hopkinson bar apparatus (SHPBs), or ultrasonic machines $[5,8,10]$.

The SHPB, which is widely used for high rate characterisation, requires assumptions about experimental conditions of alignment, stress equilibrium, and inertia [8, 1114]. For this reason, it is not always certain that the observed responses really express the intrinsic material properties at high strain rate $[14,15]$. In addition, conventional high rate techniques only give average specimen properties. In order to obtain full-field measurements, as well as to identify the accuracy of conventional high-rate data, optical techniques using high-speed cameras, combined with, e.g. photoelasticity, speckle or grid techniques, may be used to obtain additional strain measurements [8].

High rates tests on polymers have been employed to characterise fracture, voiding and cracking behaviours [16]; rate dependence due to the glass transition [7]; and strain-crystallization during deformation [17]. Challenges in data interpretation include non-constant strain rate, oscillations in the incident pulse, inhomogeneous stress and strain, non-isothermal deformation, and impedance mismatch between the specimens and loading system [1821]. From a material point of view, issues such as low magnitude transmitted signals, stress non-equilibrium and inertial effects associated with characterization of soft materials start to appear when the strength is less than $10 \mathrm{MPa}$ [22]. The difficult measurements for 'soft' materials at high strain rate have led to significant effort to obtain precise results. Authors have made developments including direct measurements using high speed photography, low impedance bar materials and thinner samples to investigate strain rates up to $10^{4} \mathrm{~s}^{-1}[9,23]$.
In this study, we calibrated the small strain temperature and frequency dependence of a commercial polyurethane, and explored large strain quasi-static compression responses, which were used to predict the high strain rate behaviour of the material. Dynamic mechanical analysis (DMA) was first executed to understand the viscoelastic behaviour as a function of temperature and frequency, producing a master curve as a function of frequency through time-temperature superposition. A numerical (FE) simulation of the DMA experiment was performed, using a Prony series fit to test data. Then, a linear viscoelastic continuum damage model based on the Boltzmann superposition integral and a strain based damage criterion was reviewed, and the model parameters were fitted to the data from these experiments. This model was further used to predict the response in cyclic tensile test and high strain rate compression experiments. The study reported in this paper thus provides an applicable methodology to characterize temperature and strain rate dependent mechanical properties of rubbers, including prediction of high rate behaviour from data obtained in low rate tests.

\section{Linear Viscoelastic Continnum Damage Theory}

Many particulate elastomer behaviours under different loading conditions can be described using phenomenological models with continuum damage criteria [24, 25]. Empirically, it is observed that with increasing strain, the stiffness of the material decreases through viscoelastic softening and changes in microstructure. Although there are many polymer-relevant physical interpretations, the issue of understanding and modelling this behaviour still exists. In this paper, a model based on viscoelasticity and continuum damage mechanics is used.

\section{Essential Viscoelastic Physical Model}

The most general models of viscoelasticity are based on physical rheology, without involving any explicit model of chemical composition or molecular variation. The models are based on mechanical analogues consisting of Hookean springs and Newtonian dashpots, and are used to establish the physical phenomenology of the viscoelasticity under investigation. A typical model consisting of multiple Maxwell elements in parallel with a spring is often used, and is shown in Fig. 1.

The stress relaxation modulus for this model can be written as

$$
E(t)=E_{0}+\sum_{i=1}^{M} E_{i} \exp \left(-\frac{t}{\tau_{i}}\right)
$$




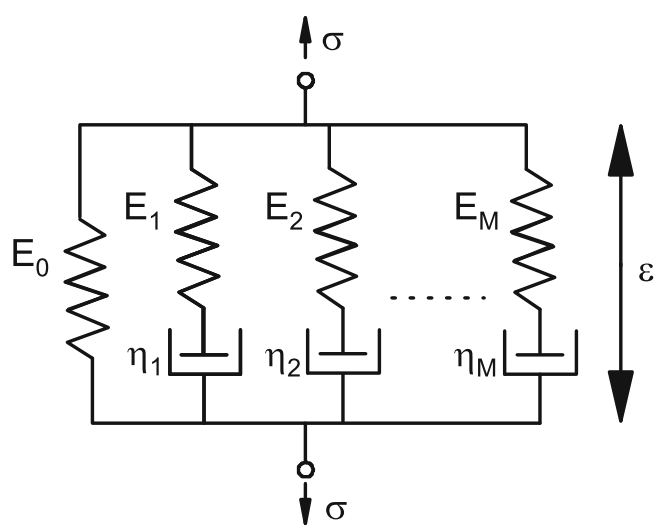

Fig. 1 Generalized Maxwell model

where $E_{i}$ denotes the elastic modulus of the spring elements; $\tau_{i}=\eta_{i} / E_{i}$ is the relaxation time of each Maxwell element.

For time varying strains, the Boltzmann superposition principle states that the incremental response of a material to a given deformation is independent of the deformation history. This is only valid in the linear viscoelastic region. Here, stress-strain relations in viscoelastic materials can be represented as the cumulative stress relaxation behaviour resulting from the entire loading history. The Boltzmann superposition allows the stress response over the loading history to be calculated by summing these relaxation results

$\sigma(t)=E(0) \varepsilon\left(0_{+}\right)+\sum_{i=0}^{M} \int_{0}^{t} E_{i} \exp \left(\frac{t-s_{i}}{\tau_{i}}\right) \frac{d \varepsilon\left(\triangle s_{i}\right)}{d s} d s_{i}$

where $s$ is a dummy variable with units of time. Here, as $t \rightarrow \infty, E(0)$ tends to the long term elastic response, i.e. $E_{0}$. If monotonic loading at a single strain rate starts at $\varepsilon=0$ and $\sigma=0$, and sufficient time has elapsed since any previous deformation, the stress-strain relation can be calculated from the corresponded strain rate and time accumulation as

$\sigma(t)=\int_{0}^{t} E(t-s) \dot{\varepsilon}(s) d s$

To apply this model of viscoelastic material properties into finite element simulations, the time domain Prony series is often used [26]. The response is assumed to be isotropic, and the Young's, shear and bulk moduli are time dependent according to

$$
\begin{aligned}
& E(t)=E(0)\left[1-\sum_{i=1}^{M} \frac{E_{i}}{E(0)}\left(1-e^{-t / \tau_{i}}\right)\right] \\
& G(t)=G(0)\left[1-\sum_{i=1}^{M} g_{i}\left(1-e^{-t / \tau_{i}}\right)\right]
\end{aligned}
$$

$K(t)=K(0)\left[1-\sum_{i=1}^{M} k_{i}\left(1-e^{-t / \tau_{i}}\right)\right]$

where $E(0), G(0)$ and $K(0)$ are the instantaneous elastic moduli in the time domain, and $g_{i}$ and $k_{i}$ are moduli ratios for each Maxwell element.

\section{Linear Viscoelastic Continuum Damage Theory}

The viscoelastic model with damage used in this paper was first introduced by Simo [27]. Continuum damage mechanics is employed to develop a simple isotropic damage mechanism, which incorporates softening behaviour under deformation and leads to a progressive degradation in storage modulus in a monotonic test with increasing amplitude. The model is driven from the free energy over any range of deformation which is uncoupled into a volumetric and deviatoric response

$\Psi(\varepsilon, q)=U^{0}(\operatorname{tr} \varepsilon)+\Psi^{0}(e)-q: e+\Psi_{I}(q)$

where $U^{0}(\operatorname{tr} \varepsilon)$ and $\Psi^{0}(e)$ are the volumetric and deviatoric stored energies, whilst $q: e$ and $\Psi_{I}(q)$ are the deviatoric and volumetric energies dissipated from the system. In particular, $\Psi_{I}(q)$ represents the volumetric energy dissipation due to viscous effects, calculated by eliminating viscous deviatoric energy from total viscous energy dissipation.

Material failure during loading is complicated: the range of structural transformations or degradation might include de-bonding, chain breakage, spherule fracture, and lamellae slippage in both micro- and macro-morphologies. In the model, all damage is summarized as an isotropic damagedominated energy dissipation leading to degradation of potential energy. The general stored energy function $\Psi(\varepsilon, D)$ for a material undergoing an isotropic damage process during loading is described as

$\Psi(\varepsilon, D)=(1-D) \Psi^{0}(\varepsilon)$

where $(1-D)$ is the damage parameter and $\varepsilon$ represents strain.

The continuum damage constitutive equation was developed to give a non-equilibrium viscous stress based on damage dissipation, expressed as

$$
\begin{aligned}
& S=\bar{g}\left(\varepsilon_{\text {Max }}\right) \frac{\partial \Psi^{0}(\varepsilon)}{\partial \varepsilon} \\
& \text { where } \varepsilon_{\text {Max }}=\max _{s \in(-\infty, t]} \sqrt{2 \Psi^{0}(\varepsilon(s))} .
\end{aligned}
$$

$\bar{g}(\varepsilon)=\beta+(1+\beta)\left(\frac{1-e^{-\varepsilon / \alpha}}{\varepsilon / \alpha}\right)$

where $S$ is the stress tensor and $\varepsilon(s)$ is the strain tensor, $\bar{g}(\varepsilon)$ represents the integrated damage parameter, empirically formulated by fitting coefficients $\alpha(\alpha \in[0, \infty))$ and $\beta$ $(\beta \in[0,1])$ [27]; $\varepsilon$ is recognized as the magnitude of true strain in a continuous loading process. 
The damage is only considered to affect the global stiffness, as a 'polymer-solid' composite morphology is assumed, rather than changing the viscous properties of the underlying 'polymer'. Hence, integrating viscoelastic behaviour through the Boltzmann superposition principle equation (4) and isotropic damage process equation (10) gives a damage model which is proportional to instantaneous stored strain energy, with empirical softening behaviour emerging from a combination of linear viscoelasticity and material damage affecting the inherent local modulus. In a monotonic test with a constant strain rate, the response can be simplified as

$\alpha(t)=\int_{0}^{t} E(t-s) \bar{g}(\varepsilon) \dot{\varepsilon}(s) d s$

\section{Method and Results}

The linear viscoelastic properties are first obtained from DMA experiments, which are integrated with quasistatic monotonic tests in compression to determine the phenomenological parameters for the damage model. The aim of this project is to investigate the whether the use of linear viscoelastic continuum damage theory in conventional mechanical experiments allows correlation between quasi-static and high strain rate tests. This is done by testing a commercial carbon-black filled TPU elastomer (TPUs-Shore-A90, Bonaprene Products Ltd. UK). Further, the paper explores prediction of the resultant stress response under tensile and cyclic loading situations.

\section{Rheological Model from DMA}

Experiments were performed on a DMA machine (TA Q800), in which a beam (sample dimension $60 \times 10 \times$ $5 \mathrm{~mm}$ ) was tested in a dual-cantilever configuration under cyclic deformation. The machine outputs complex modulus ( $E^{\prime}$ and $\left.E^{\prime \prime}\right)$, loss tangent $(\tan \delta$ ), stress, temperature and frequency, the first four of which are calculated from the amplitude and phase of the applied displacement and resultant force. Isothermal frequency sweeps were performed at $2{ }^{\circ} \mathrm{C}$ intervals between $-48{ }^{\circ} \mathrm{C}$ and $80{ }^{\circ} \mathrm{C}$, and frequencies $0.5,2,5$ and $10 \mathrm{~Hz}$. The displacement amplitude was $30 \mu \mathrm{m}$. From the output values of moduli against loading frequency at each temperature, a master curve of frequency dependence was produced using timetemperature superposition.

Figure 2 shows the dependence of moduli on temperature at the four different frequencies. For each temperature, it is also possible to plot modulus as a function of frequency, and then to shift these horizontally to produce a master curve of modulus against frequency at a chosen reference temperature (Fig. 3).

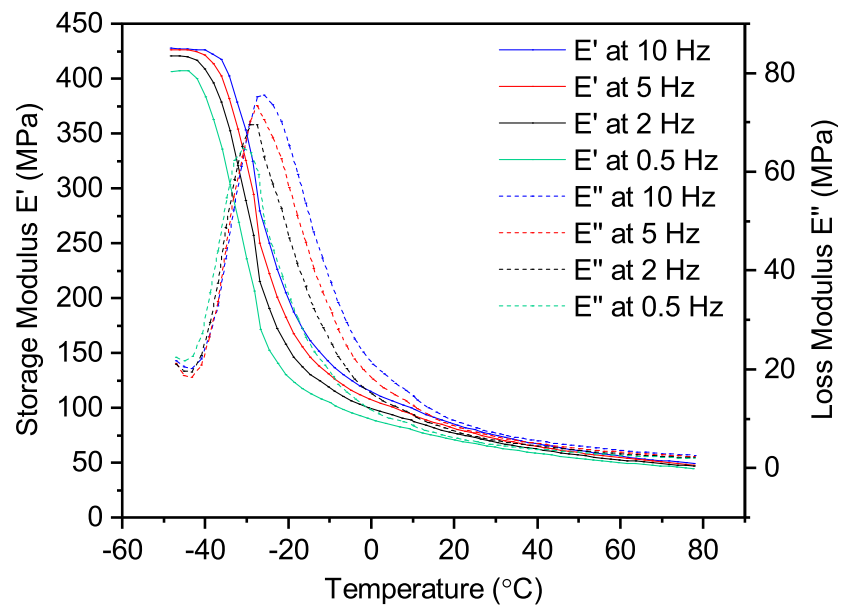

Fig. 2 Moduli $E^{\prime}$ and $E^{\prime \prime}$ as a function of temperature: DMA data obtained from isothermal frequency sweeps at four frequencies: $0.5,2$, 5 and $10 \mathrm{~Hz}$

Figure 4 depicts plots of shift factor vs. temperature at two reference temperatures. Two approximately tangential lines at high and low temperature can be fitted to the curves; these intersect at the same point on the temperature axis. This intersection point indicates that the polyurethane has glass temperature, $T_{g}$, of about $-27^{\circ} \mathrm{C}$. For interest, it is also shown that the WLF formula equation (1) fitted the empirical data around this point.

The plot of moduli against frequency obtained from TTS can further be described by the Generalized Maxwell Model

$E^{\prime}(\omega)=E_{0}+\sum_{i=1}^{M} E_{i} \frac{\tau_{i}{ }^{2} \omega^{2}}{1+\tau_{i}{ }^{2} \omega^{2}}$

$E^{\prime \prime}(\omega)=\sum_{i=1}^{M} E_{i} \frac{\tau_{i} \omega}{1+\tau_{i}{ }^{2} \omega^{2}}$

$\tan \delta=\frac{E^{\prime \prime}}{E^{\prime}}$

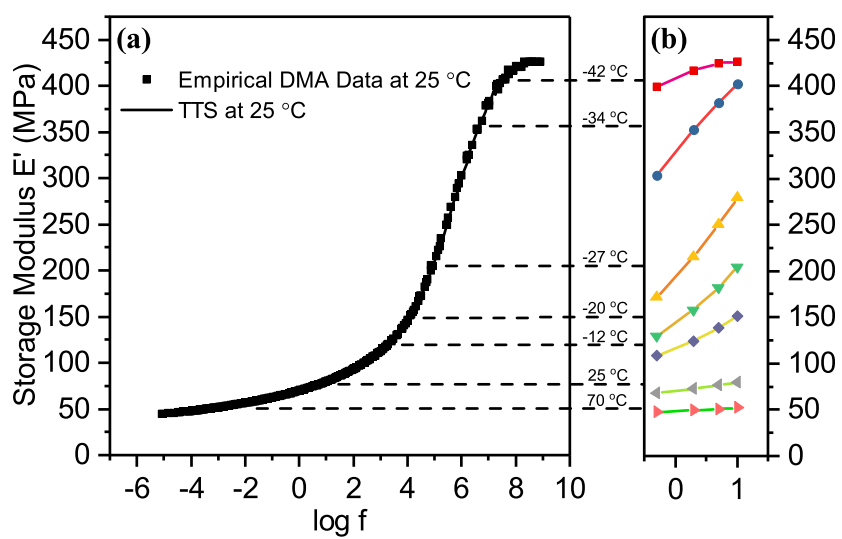

Fig. 3 TTS applied to $E^{\prime}-\log f$ plots from DMA experiments: (a) master curve of $E^{\prime}-\log f$ at $25^{\circ} \mathrm{C}$ and (b) $E^{\prime}-\log f$ plots at selected temperatures, as indicated in the figure 


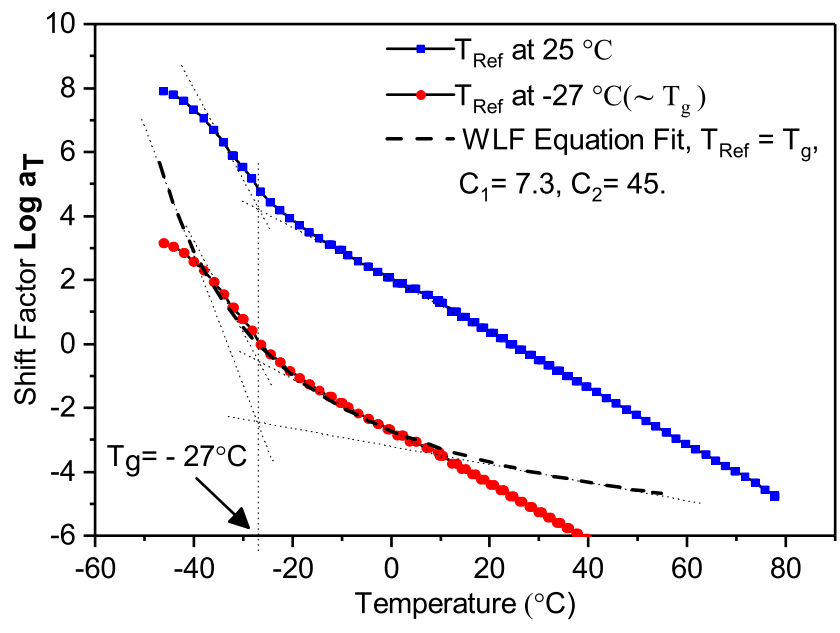

Fig. 4 Shift factor vs. temperature at reference temperatures of $25^{\circ} \mathrm{C}$ and $-27{ }^{\circ} \mathrm{C}$; WLF equation evaluated at $T_{g}=-27^{\circ} \mathrm{C}$ with parameters $C_{1}=7.3^{\circ} \mathrm{C}, C_{2}=45^{\circ} \mathrm{C}$

where $\omega$ is the angular frequency of the applied deformation, and $E_{0}, E_{i}$ and $\tau_{i}$ are the properties of elements from the Generalized Maxwell Model. The storage and loss moduli are defined as the real and imaginary parts of the complex modulus during the dynamic harmonic cyclic loading. The phase shift factor $\tan \delta$ is the ratio of loss modulus to storage modulus and represents the phase lag between strain and stress responses.

Figures 5 and 6 show the superposition results for storage, $E^{\prime}$, and loss, $E^{\prime \prime}$, moduli, obtained from TTS at a reference temperature $25{ }^{\circ} \mathrm{C}$. The next step is to use
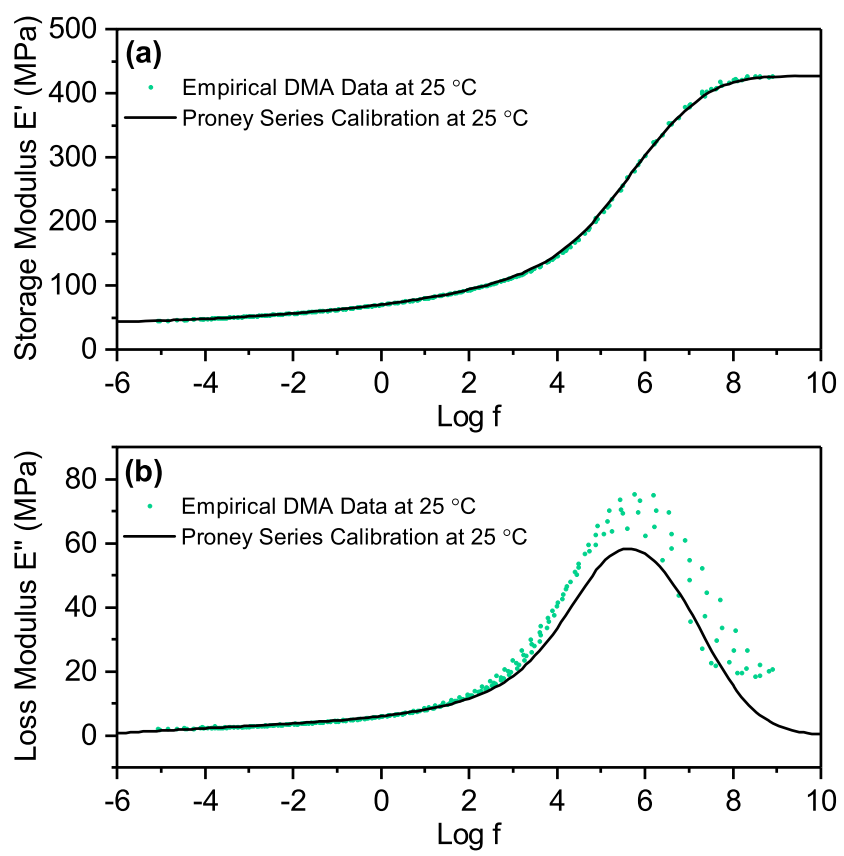

Fig. 5 (a) Storage modulus $E^{\prime}$, (b) Loss modulus $E^{\prime \prime}$ vs. $\log f$ showing Prony series fit
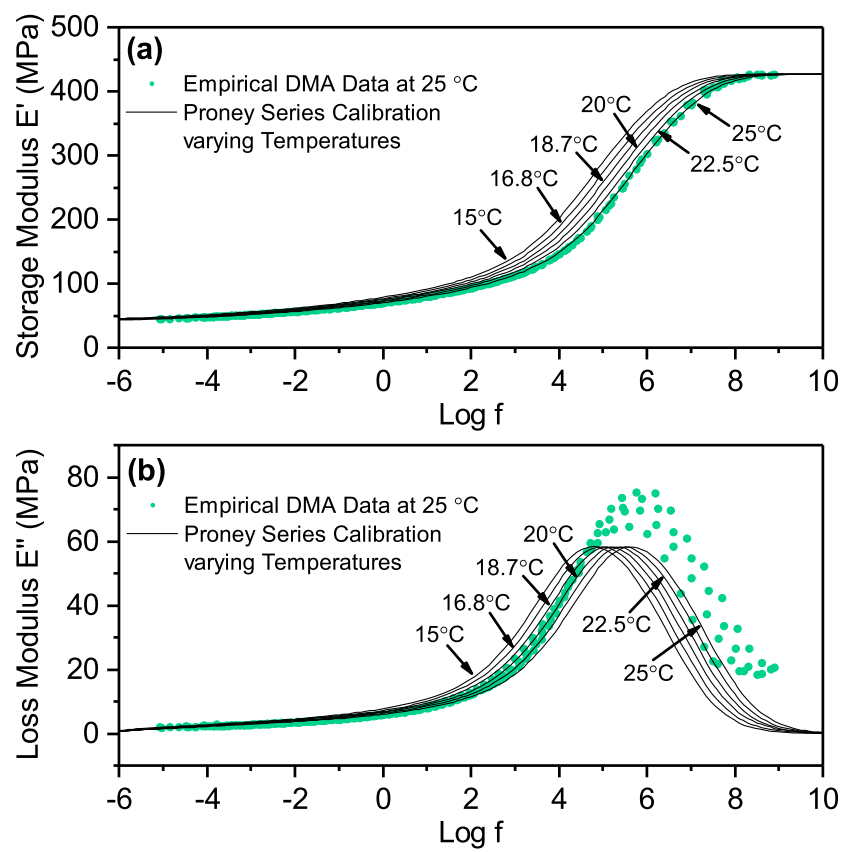

Fig. 6 (a) Storage modulus $E^{\prime}$, (b) Loss modulus $E^{\prime \prime}$ vs. $\log f$ showing Prony series fit for various reference temperatures

the Alfrey Approximation, a method to switch between relaxation measurements and retardation time spectra to parameterise the Prony series for the Generalized Maxwell Model [28]. Further calculation of Prony series is given in Supplementary Information. It was noted that a single set of Prony series parameters could not fit both the storage and loss moduli accurately, this is likely to be a result of experimental error in the loss values, which are an order of magnitude smaller than the storage. However, it will be seen later that fitting the series to the storage modulus produced a model which agreed well with later compression experiments. The Prony series was then implemented in the model calibration of quasi-static experiments in equation 12.

The Prony series was then implemented into FE simulation software (Abaqus FEA) and analytical and FE models of the DMA experiment were performed to confirm the reliability of DMA results; a description of the analytical beam model and the FE material model validation are given in Supplementary Information. The models for the DMA used the same frequencies, specimen dimensions and deflection magnitude as the real experiment. The FE model was meshed using C3D8I elements, with 10 elements through the thickness of the specimen. An oscillating deflection was applied to the surfaces of the beam centre to model the central DMA clamp. The side clamps in the DMA were simulated by using fixed (ENCASTRE) boundary conditions. A picture of the model is shown in Fig. 7, whilst the force vs. time results are shown in Fig. 8, these show good agreement between the models and the DMA 
Fig. 7 Numerical model of DMA experiment using dual cantilever configuration

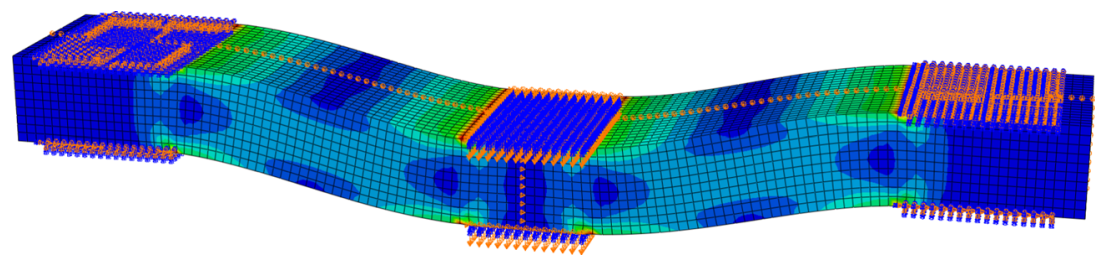

output data, confirming that the tests and derived material properties are consistent.

\section{Phenomenological Damage Model from Quasi-Static Compression Experiments}

Quasi-static experiments were used to calibrate the damage model. Tests with monotonic and cyclic loading were executed in a commercial screw-driven testing machine (Instron 5982). For tensile tests, strips (dimension of 5 $\times 10 \times 200 \mathrm{~mm}$ ) were used, whilst in compression, cylinders (dimension of $5 \mathrm{~mm}$ diameter $\times 5 \mathrm{~mm}$ length) were tested (a full description of the experiment apparatus is given in Supplementary Information). The monotonic tension and compression experiments were performed in true-strain control at target strain rates of $0.1,0.01$ and $0.001 \mathrm{~s}^{-1}$. The resulting force was recorded by a load cell, whilst strain was directly measured: in tension a travelling extensometer was attached to the specimen; in compression a contacting extensometer was used attached to the loading anvils near to the specimen. The digital image correlation (DIC) technique was also applied to confirm the strains obtained from the extensometers. DIC is a standard technique to obtain full field deformation data by tracking the movement of a random pattern on the specimen surface. Here, a camera (PointGrey Ltd.) was used to capture images of the specimen during tests, and commercial DIC software (DaVis V8) was used to analyse the images. The frame rate
Fig. 8 Comparison of analytical and FE model outputs with DMA experiment: (a) Full range of frequencies; (b) $10 \mathrm{~Hz}$; (c) 5 $\mathrm{Hz}$; (d) $2 \mathrm{~Hz}$; (e) $0.5 \mathrm{~Hz}$
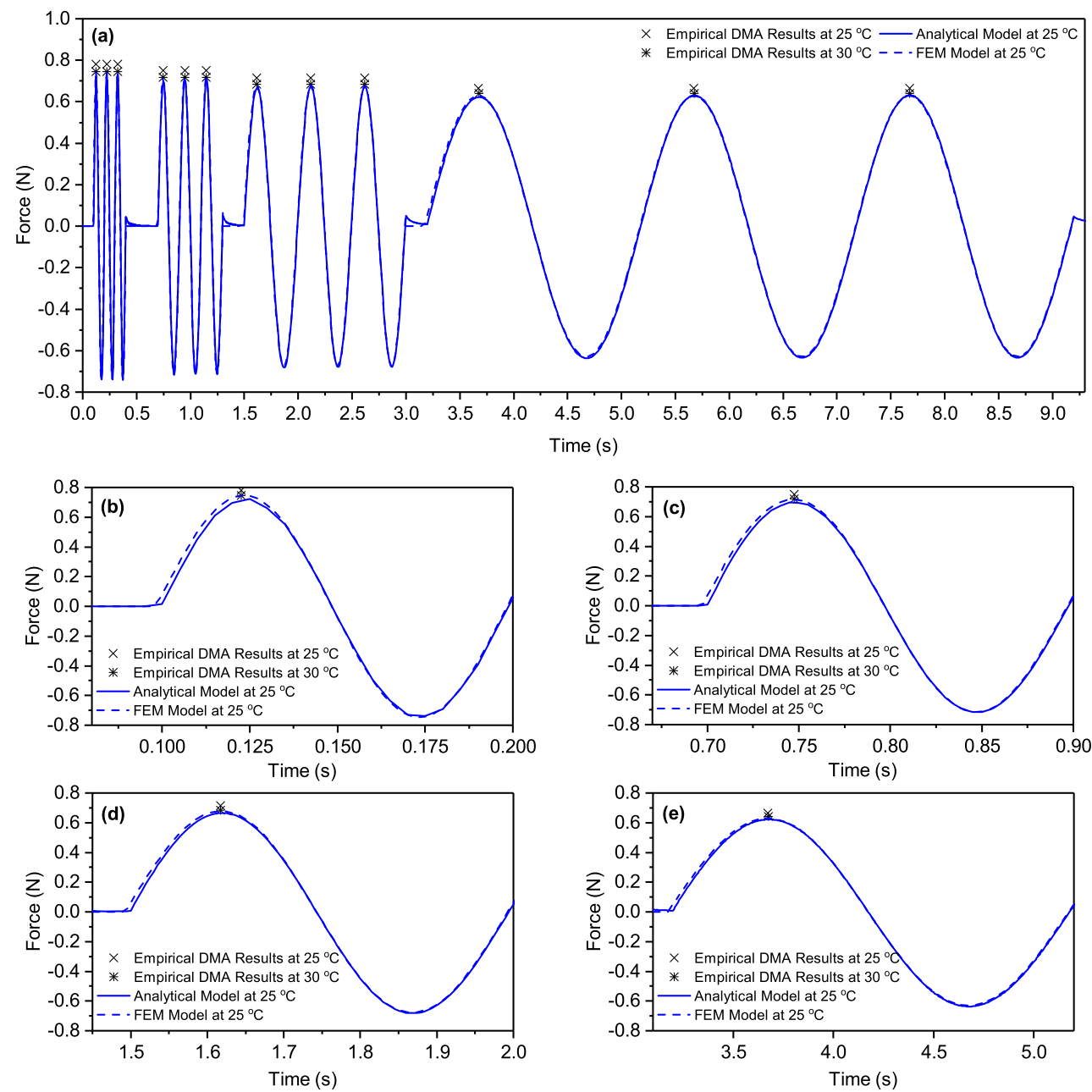
was set as $5 \mathrm{fps}$ and an image of an undeformed specimen was set as the reference. The pattern was applied using spray paint. The DIC analysis results showed that the constant true strain rates achieved were $0.08,0.008,0.0008 \mathrm{~s}^{-1}$ in tension; and 0.09, 0.009 and $0.0009 \mathrm{~s}^{-1}$ in compression. The maximum strains used were limited by slipping in the clamp in the tensile experiments.

In general linear viscoelastic continuum damage theory, the damage is mainly dependent on the strain magnitude, independent of the change in loading rate and loading direction [27, 28]. A comparison between 'inherent' and empirical local stiffness is used to introduce a damage factor $\bar{g}(\varepsilon)$ which represents the magnitude of phenomenological softening. The calibration of material softening was first executed by comparing stress-strain responses generated from DMA data using the Boltzmann superposition principle, to stress-strain data generated in the compression and tension experiments. Initially, compression data were used: Fig. 9(a) shows the empirical compression experiments compared to 'inherent' stressstrain curves obtained from the Boltzmann superposition integral. Figure 9(b) compares the local stiffnesss from the experiments to those from the Boltzmann superposition integral. The damage factor, calibrated by optimising $\alpha$ and $\beta$ in equation (11) in Fig. 9(c), is successfully fitting the ratio of stiffness in the empirical data. It should be noted that at each strain rate at least three experiments were performed and the curves shown are chosen from the experiment closest to the mean of the data: the full set of data and model simulation outputs are shown in Supplementary Information.

The damage model was compared to experiments at different strain rates (Fig. 10(a)) and temperatures (Fig. 10(b)) separately. These comparisons demonstrate that the model is able to reproduce very accurately the compressive responses with a single set of $\alpha$ and $\beta$ parameters.

\section{Application in Quasi-Static Tensile Cyclic Tests}

The ambient temperature for the tensile experiments was $16.8{ }^{\circ} \mathrm{C}$, so the Boltzmann superposition integral was recalculated at this temperature for three rates: 0.08, 0.008 and $0.0008 \mathrm{~s}^{-1}$. Cyclic tensile tests with a single loop were executed first: at least three experiments were conduced at each rate, with virgin samples used each time (i.e. nine fresh samples were tested in total). The full set of data is presented in Supplementary Information. Figure 11(a) shows the resulting local stiffnesses from the Boltzmann superposition integrals and one experiment at each rate. These are compared to calculate the damage factors in Fig. 11(b).
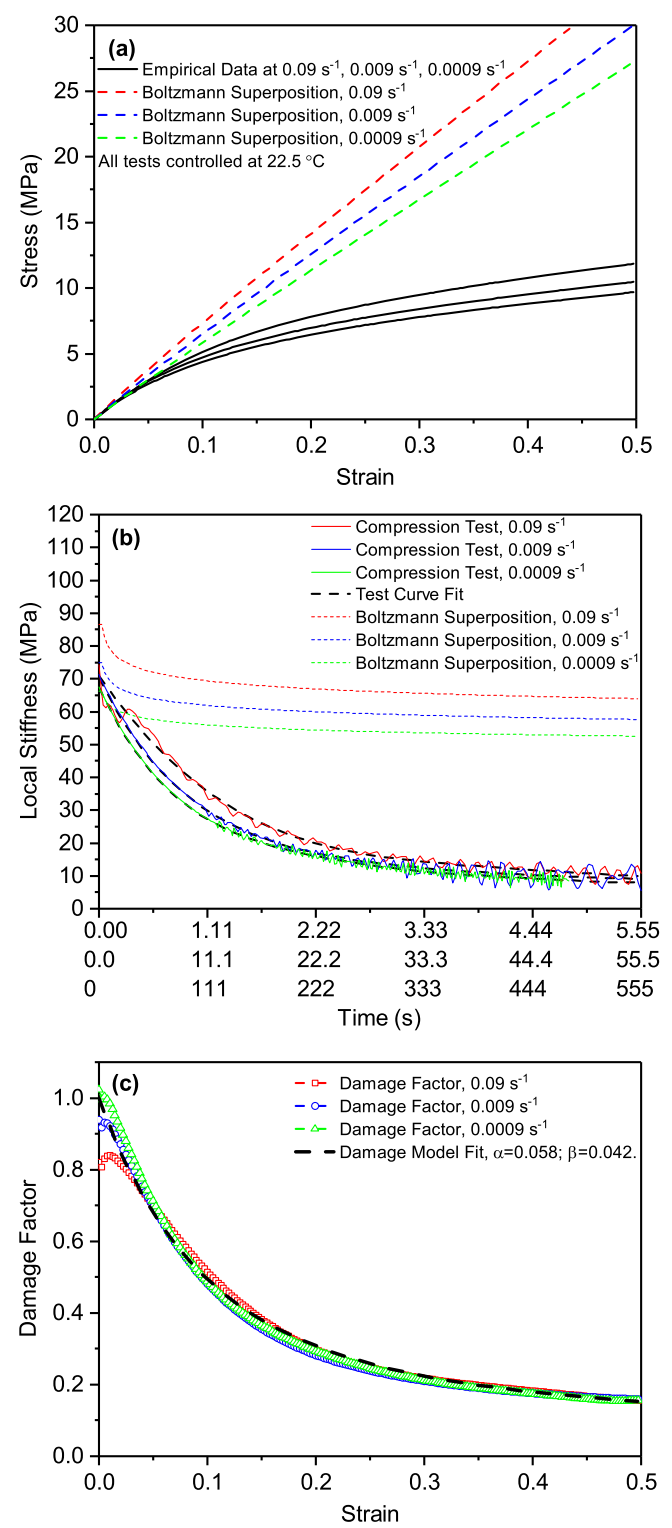

Fig. 9 The process to determine the phenomenological damage factor: (a) stress-strain curves from compression experiments and Boltzmann superposition principle at three strain rates, $22.5^{\circ} \mathrm{C} ;(\mathbf{b})$ comparison of local stiffnesses from experiments and Boltzmann superposition; (c) the parameters $\alpha=0.058, \beta=0.042$ for the phenomenological damage factor (i.e. the ratio of the stiffnesses in $(\mathbf{b})$ ) were determined from the average of three strain rates: $0.09 \mathrm{~s}^{-1}, 0.009 \mathrm{~s}^{-1}$ and $0.0009 \mathrm{~s}^{-1}$ by fitting equation (11) to the empirical data. The damage model was calibrated at $22.5^{\circ} \mathrm{C}$

Here it is noted that the experimentally calculated damage factor exceeds 1 . This is probably the result of experimental errors at small stains, in particular measurements of the strains themselves, the calculated damage factor $\bar{g}(\varepsilon)$ cannot exceed 1. It is also noted here that the factors jump on the switch from loading to unloading. Whilst some of the jump in stiffness is accounted for in the viscoelastic response (from the Boltzmann superposition integral) this does not 

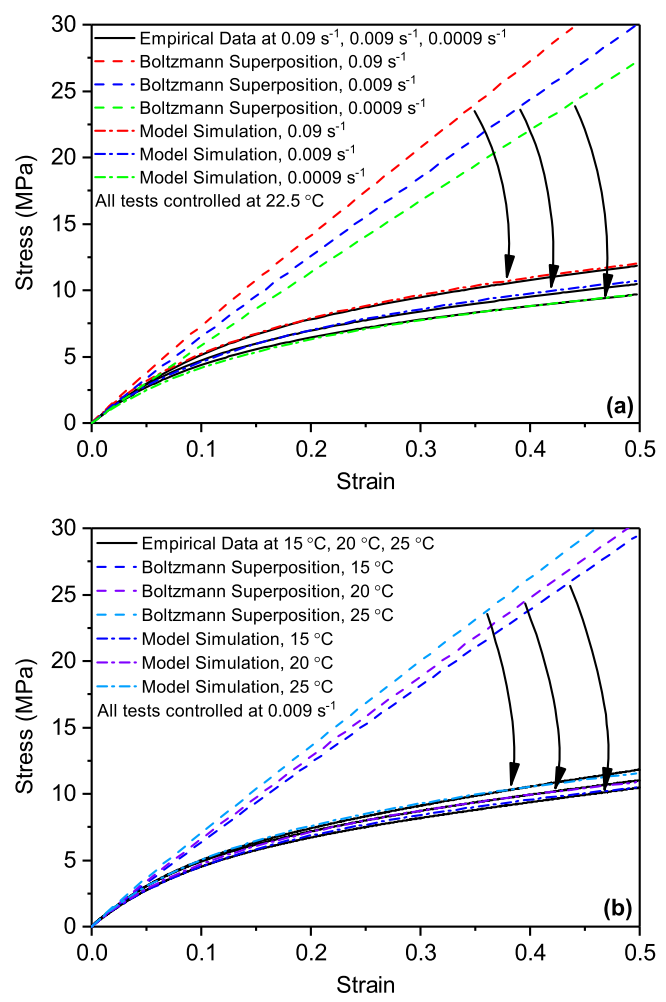

Fig. 10 Comparison of experimental data to model predictions when varying strain rates (a) or temperatures (b) in compression experiments. The damage parameters from Fig. $9(\alpha=0.058$, $\beta=0.042$ ) were used. The rate and temperature effects were captured using appropriate TTS master curves (Fig. 6) and the Boltzmann superposition integral equation (12)

give a full description of the overall material response. As a result, the implementation of the viscoelastic continuum damage was changed so that the damage parameters, $\alpha$ and $\beta$, are defined as strain-dependent only but also that the 'strain' is reset at the start of the each cycle, i.e. each time the direction of loading is reversed. The output from this model is then shown in Fig. 11(c).

An additional study was performed in which repeated cyclic tests were executed at $0.008 \mathrm{~s}^{-1}$ strain rate. Here a single sample was subjected to a large number of cycles in order to assess the damage accumulation. The results (stress-strain curve and damage factor only) are shown in Fig. 12, where they are compared to the model used up to this point. A possible improvement to the model is to redefine the parameters during loading. Figure 12 shows that the damage parameters through the entire cyclic loading can be classified into two groups: one for the initial loading process and a second one for the remaining cases. By controlling the second set of parameters, the model was much better able to describe the experiment, consistent with the so-called 'Mullins effect' and the softening observed during the cyclic loading. In particular, the Mullins effect is the name given to a loss of stiffness that can be
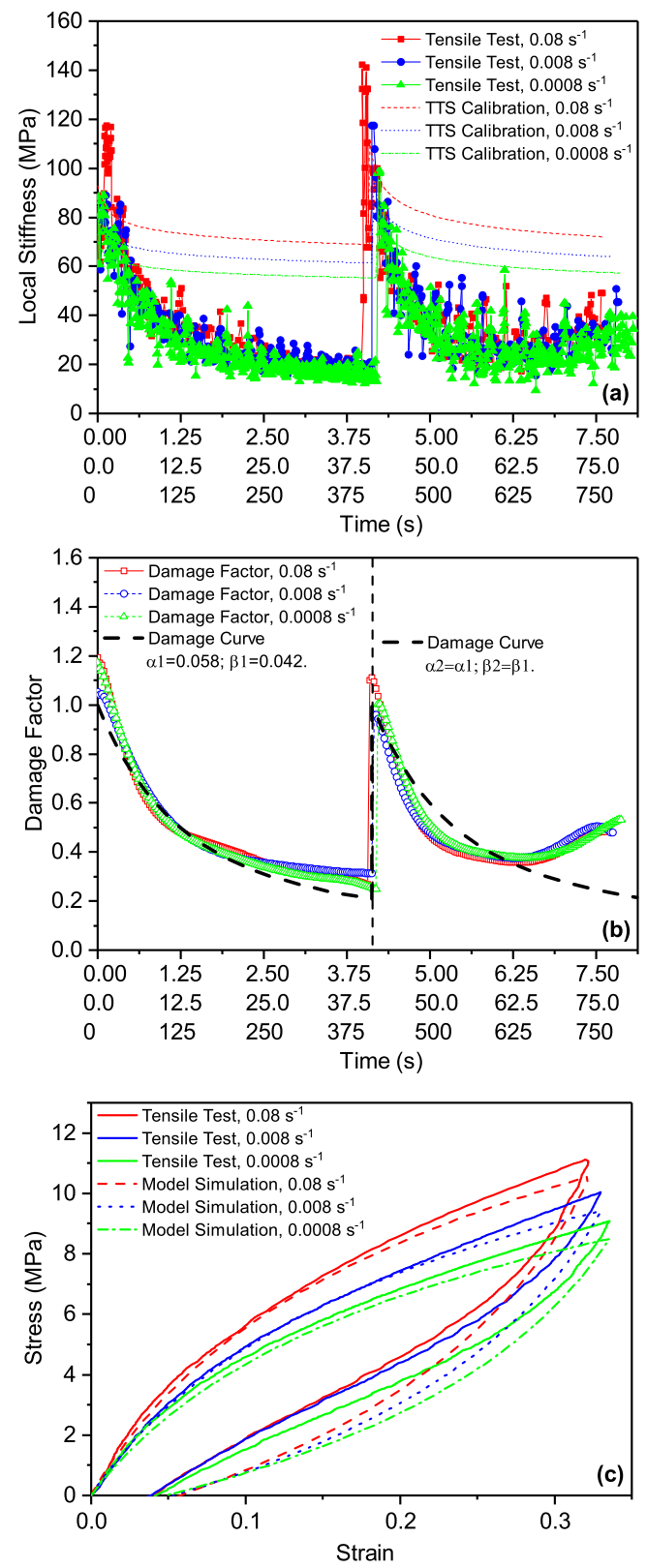

Fig. 11 Data obtained in cyclic tensile experiments with constant temperature of $16.8^{\circ} \mathrm{C}$ but varying strain rates of $0.08 \mathrm{~s}^{-1}, 0.008 \mathrm{~s}^{-1}$ and $0.0008 \mathrm{~s}^{-1}$. The local stiffnesses from the Boltzmann superposition integral and experiments are plotted, (a), to compare the damage parameters $\alpha=0.058, \beta=0.042$ from compression in Fig. 9(c) to the empirical ratio from tensile experiments, (b). The same parameters are adopted with strain reset at the start of the unloading process, to produce the simulation, $(\mathbf{c})$, at $16.8{ }^{\circ} \mathrm{C}$

correlated to the maximum stress achieved in recent prior loadings, but which recovers over time. This is often observed, in particular, in filled materials. Various physical interpretations have been given [24], including cavities, chain session and disentanglement contributing to damage within the rubber matrix [29-31]. 
Fig. 12 A multi-cyclic tensile experiment was executed at $16.8^{\circ} \mathrm{C}$ and $0.008 \mathrm{~s}^{-1}$ strain rate. The existing parameters of $\alpha=0.058, \beta=0.042$, (a), were used for the entire model, (b); while the switchable parameters: $\alpha_{1}=0.058, \beta_{1}=0.042$ for the primary loading, and $\alpha_{2}=0.012$, $\beta_{2}=0.417$ for the remaining, (c), exhibits better results, (d)


\section{Application to Split-Hopkinson Bar Experiment}

The split Hopkinson bar technique is well developed and is now almost a standard method to study the high rate compression of materials $[8,32]$. In the experiments implemented here, a cylindrical specimen (of $5 \times 5 \mathrm{~mm}$ ) was placed between the ends of two magnesium alloy bars, Fig. 13. A smaller 'striker' bar is accelerated towards the incident bar by mean of pressurised gas. This creates a stress wave which travels down to the specimen, the incident wave. At the bar-specimen interface, a portion of the input wave is reflected and the rest transmitted. The incident, reflected and transmitted waves are measured by strain gauges attached to the bars. These stresses can be used to calculate the deformation rate and force in the specimen, which can be converted to stress and strain.

Specifically, the strain rate in the specimen is given by

$\frac{d \varepsilon}{d t}=\frac{c}{l_{S}(t)}\left[\varepsilon_{i}(t)-\varepsilon_{r}(t)-\varepsilon_{t}(t)\right]$

where $\varepsilon_{i}(t), \varepsilon_{r}(t)$ and $\varepsilon_{t}(t)$ are the measured strain pulses, $l_{s}(t)$ is the specimen length and $c=\sqrt{E / \rho}$ is the longitudinal wave-speed in the bar. The stress is given by

$\sigma(t)=\frac{A_{b}}{A_{s}} E \varepsilon_{t}(t)$

where $A_{b} / A_{s}$ is the ratio of cross-sectional area of the transmission bar and the specimen. The key assumption of this analysis is that the specimen is in static equilibrium (i.e. the stress is the same along its length), which in practice means that

$\varepsilon_{i}=-\varepsilon_{r}+\varepsilon_{t}$

In order to achieve this, higher strain rates require progressively thinner samples, and indeed this may be difficult or impossible to achieve in specimens manufactured from low wave-speed materials, and must be checked experimentally.

Here, the compression SHPB experiments up to $2000 \mathrm{~s}^{-1}$ were executed. The linear viscoelastic continuum damage model was used to predict high strain rate compression data, again using the master curve obtained from DMA experiments. Figure 14 shows comparison of the model and experimental data at $18.7^{\circ} \mathrm{C}$.

Two additional factors must be taken into account when interpreting data from Hopkinson bar experiments. The
Fig. 13 Experimental configuration for SHPB test

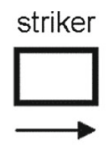

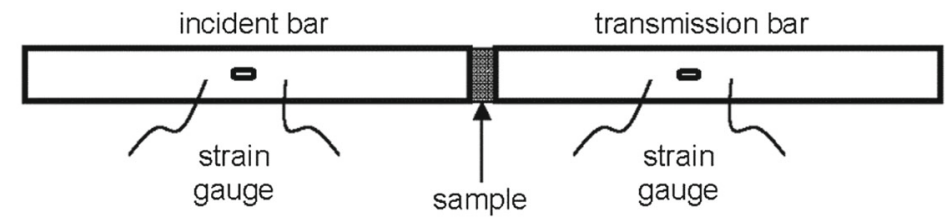



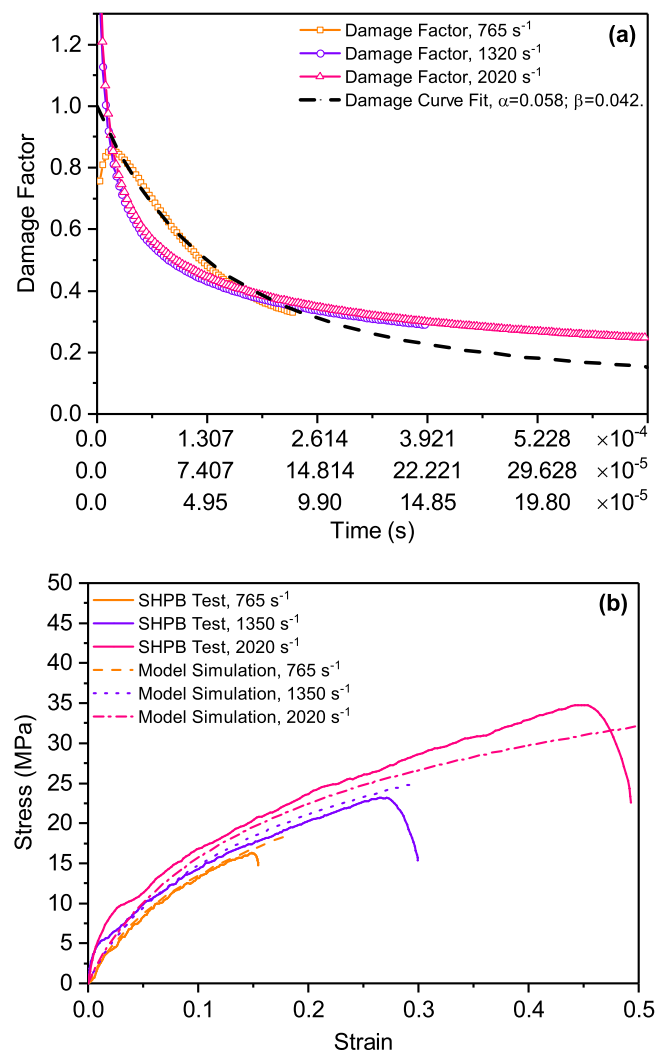

Fig. 14 High strain rate compression experiments were executed at $18.7{ }^{\circ} \mathrm{C}$ at three strain rates: $765 \mathrm{~s}^{-1}, 1350 \mathrm{~s}^{-1}$ and $2020 \mathrm{~s}^{-1}$. The existing parameters of $\alpha=0.058, \beta=0.042$, (a), were used again for the model simulation $(\mathbf{b})$

first is the effect of specimen inertia, owing to the rapid acceleration of the specimen during the experiments. This adds to the stress measured in the system. The specimen is initially at rest, and is accelerated to the peak strain rate $2020 \mathrm{~s}^{-1}$. During this acceleration, inertia-induced stress is encountered in both axial and transverse directions, which add to the inherent material response [33]. The specimen stress measured during a Hopkinson bar experiment may therefore be through of as the sum of stress components [33]:

$\sigma_{T}=\sigma_{M}+\sigma_{I}+\sigma_{F}+\sigma_{o t h e r}$

where $\sigma_{T}$ is the stress measurement, $\sigma_{M}$ is the material strength to be measured, $\sigma_{I}$ is an enhancement owing to inertia, $\sigma_{F}$ is an enhancement owing to friction and $\sigma_{\text {other }}$ is the enhancement owing other effects that may be present.

The additional inertia stress can be calculated for an incompressible material as [34]

$\sigma_{I}=\rho \ddot{\varepsilon}\left(\frac{a^{2}}{8}-\frac{h^{2}}{6}\right)+\rho \dot{\varepsilon}\left(\frac{a^{2}}{16}+\frac{h^{2}}{6}\right)-\frac{\rho h \dot{v}}{2}$

where $\rho$ is the material density, $\dot{\varepsilon}$ and $\ddot{\varepsilon}$ are strain rate and strain acceleration, $a \approx 2.5 \mathrm{~mm}$ is the radius, and $h \approx 5 \mathrm{~mm}$ is the height for this specimen, and $v$ represents the speed of the output bar. The magnitude of this inertia stress is only $1700 \mathrm{~Pa}$ at a strain rate of $2020 \mathrm{~s}^{-1}$, so that the inertia effect is not significant in these experiments.

The second potential effect is the temperature rise owing to the conversion of mechanical work to heat. The high strain rate experiment is assumed to take place adiabatic conditions. This means that any heat generated during the loading will be retained in the specimen, causing an increase in temperature in equations (21) and (22), which can be approximately assuming that all the mechanical work done is converted to heat by [35].

$$
\begin{aligned}
& \Delta Q \approx \Delta W \rightarrow \int_{0}^{\varepsilon} \sigma d \varepsilon=\rho C_{p} \Delta T \\
& \Delta T(\varepsilon)=\frac{\beta}{\rho C_{p}} \int_{0}^{\varepsilon} \sigma d \varepsilon
\end{aligned}
$$

where $\Delta W$ is the work done, $\Delta Q$ is the heat generated, $\sigma$ the true stress, $\varepsilon$ the true strain, $\rho=1000 \mathrm{~kg} \mathrm{~m}^{-3}$ the material density, $C_{p}=1800 \mathrm{~J} \mathrm{~kg}^{-1} \mathrm{~K}^{-1}$ the specific heat capacity at constant volume and $\Delta T$ the rise in temperature. Here, material properties have been obtained from the supplier, Bonaprene Products Ltd., UK.

Using the assumption that all the work is converted to heat, which is a significant over-estimate for these materials in which we have shown the that quasi-static loading is recoverable, this equation gives a temperature rise of only $0.34{ }^{\circ} \mathrm{C}$ at $2020 \mathrm{~s}^{-1}$, which can be ignored.

\section{Discussion and Conclusions}

A viscoelastic constitutive model with effects of continuum damage has been shown to provide an accurate characterization of the mechanical behaviour of thermoplastic polyurethane under uniaxial loading from low to high strain rates. A specific viscoelastic damage model for the mechanical behaviour was used and, and when the phenomenological damage criteria were obtained from quasi-static compression experiments, this model was able to predict behaviour in compression at different (quasi-static) rates and temperatures, (quasi-static) tension and high rate compression.

The material used, a commercial thermoplastic polyurethane, showed a strong dependence on both temperature and frequency, which was calibrated using DMA experiments. The TTS principle was used to produce a master curve from which a Prony series was calibrated to describe the viscoelastic material behaviour. Good results were obtained by using the linear viscoelasticity model thus obtained in numerical and finite element models of the DMA experiments over the full range of frequencies. 
The observed material softening resulted from material damage was then investigated. The damage was observed to depend on strain magnitude and was characterised by a two parameter model. By combining this with the Boltzmann superposition integral (for rate and temperature effects), both temperature and rate dependences of the stress-strain response could be simulated. Having calibrated the model using compression data, there is a small difference between this and the tensile results, which may be due to experimental effects, including specimen variability, load application (e.g. frictional boundary in compression) and uneven stress distributions in the deforming specimen. In addition, whilst the model shows good agreement with the cyclic loading data, it was observed that two sets of damage factors improve the fit; this is consistent with damage introduced in the first cycle affecting subsequent results, i.e. with the Mullins effect, although the response under cycles with increased amplitude was not measured in this study. The cyclic loading process was modelled through a combination of the Boltzmann superposition integral combined with the strain dependent damage factor; when calculating the strain dependent damage, the strain itself was 'reset' at each reversal of direction. Figures $11 \& 12$ for cyclic experiments revealed that the switch of factor parameters on reversal of the direction of loading is effective in simulating the observed response, which indicates loading direction probably does not change the damage criteria in a continuous cyclic process. Finally, the model was shown to predict experimental data in high strain rate experiments. This is significant, as it gives further validation for the use of time-temperature superposition based approaches to predict high rate response. Such indirect methods are increasingly important as we seek to obtain high strain rate data from materials with very low stiffness and sound speeds. Further work is necessary to fully account for the small differences in damage model between compression and tension, and to explore cyclic loading with different amplitudes. Better understanding and characterisation of filler structure will allow better investigation of the local stiffness degradation and hysteresis behaviours, whilst further validation of the implementation at different strain rates would also give further confidence in this approach.

Acknowledgements This material is based upon work supported by the Air Force Office of Scientific Research, Air Force Material Command, USAF under Award No. FA9550-15-1-0448. We thank Maj M Snyder and Dr. J Foley for their continued support. We also thank Nick Hawkins from the University of Oxford who provided insight and expertise that greatly assisted the polymer research, and Igor Dyson, Richard Duffin and Wolfgang Mix for help with the experimental programme and specimen preparation.

Funding This material is based upon work supported by the Air Force Office of Scientific Research, Air Force Material Command, USAF under Award No. FA9550-15-1-0448.

\section{Compliance with Ethical Standards}

Conflict of interests The authors declare that they have no conflict of interest.

Open Access This article is licensed under a Creative Commons Attribution 4.0 International License, which permits use, sharing, adaptation, distribution and reproduction in any medium or format, as long as you give appropriate credit to the original author(s) and the source, provide a link to the Creative Commons licence, and indicate if changes were made. The images or other third party material in this article are included in the article's Creative Commons licence, unless indicated otherwise in a credit line to the material. If material is not included in the article's Creative Commons licence and your intended use is not permitted by statutory regulation or exceeds the permitted use, you will need to obtain permission directly from the copyright holder. To view a copy of this licence, visit http:// creativecommonshorg/licenses/by/4.0/.

\section{References}

1. Prisacariu C (2011) Polyurethane elastomers: from morphology to mechanical aspects. Springer Science \& Business Media

2. Buckley C, Prisacariu C, Martin C (2010) Elasticity and inelasticity of thermoplastic polyurethane elastomers: Sensitivity to chemical and physical structure. Polymer 51:3213-3224

3. Kojio K, Furukawa M, Nonaka Y, Nakamura S (2010) Control of mechanical properties of thermoplastic polyurethane elastomers by restriction of crystallization of soft segment. Materials 3:50975110

4. Jiang L, Wu J, Nedolisa C, Saiani A, Assender HE (2015) Phase separation and crystallization in high hard block content polyurethane thin films. Macromolecules 48:5358-5366

5. Ferry JD (1980) Viscoelastic properties of polymers, John Wiley and Sons

6. Williams ML, Landel RF, Ferry JD (1955) The Temperature Dependence of Relaxation Mechanisms in Amorphous Polymers and Other Glass-forming Liquids. J Am Chem Soc 77(14):37013707

7. Roland C (2006) Mechanical behavior of rubber at high strain rates. Rubber Chem Technol 79:429-459

8. Field JE, Walley SM, Proud W, Goldrein H, Siviour C (2004) Review of experimental techniques for high rate deformation and shock studies. Int J Impact Eng 30:725-775

9. Siviour CR (2017) High strain rate characterization of polymers. AIP Conference Proceedings, p 060029

10. Sarva SS, Deschanel S, Boyce MC, Chen W (2007) Stress-strain behavior of a polyurea and a polyurethane from low to high strain rates. Polymer 48:2208-2213

11. Gorham D, Pope P, Field JE (1992) An improved method for compressive stress-strain measurements at very high strain rates. Proc R Soc London Series A: Math Phys Sci 438:153-170

12. Chen W, Zhang B, Forrestal M (1999) A split Hopkinson bar technique for low-impedance materials. Exper Mech 39:81-85

13. Dioh N, Leevers P, Williams J (1993) Thickness effects in split Hopkinson pressure bar tests. Polymer 34:4230-4234

14. Chen W, Song B (2010) Split Hopkinson (Kolsky) bar: design testing and applications. Springer Science \& Business Media

15. Siviour CR, Jordan JL (2016) High strain rate mechanics of polymers: a review. J Dyn Behav Mater 2:15-32

16. Fan J, Weerheijm J, Sluys L (2015) High-strain-rate tensile mechanical response of a polyurethane elastomeric material. Mater Des 79:73-85 
17. Zhao B, Tian N, Liu Y, Yan T, Zhou W, Li L, Zhou Y, Weng G, Huang G (2012) Strain-induced crystallization of natural rubber with high strain rates. J Polym Sci Part B: Polym Phys 50:16301637

18. Chen W, Lu F, Frew D, Forrestal MJ (2002) Dynamic compression testing of soft materials. Appl Mech 69:214-223

19. Song B, Chen W (2004) Dynamic stress equilibration in split Hopkinson pressure bar tests on soft materials. Exper Mech 44:300-312

20. Chen W, Lu F, Zhou B (2000) A quartz-crystal-embedded split Hopkinson pressure bar for soft materials. Exper Mech 40:1-6

21. Kendall MJ, Siviour CR (2013) Experimentally simulating adiabatic conditions: approximating high rate polymer behavior using low rate experiments with temperature profiles. Polymer 54:5058-5063

22. Chen W (2016) Experimental methods for characterizing dynamic response of soft materials. J Dyn Behav Mater 2:2-14

23. Yoon S-H, Siviour CR (2017) Application of the virtual fields method to rubbers under medium strain rate deformation using both acceleration and traction force data. Strain 53:e12225

24. Diani J, Fayolle B, Gilormini P (2009) A review on the Mullins effect. Eur Polym J 45:601-612

25. Park SW, Kim YR, Schapery RA (1996) A viscoelastic continuum damage model and its application to uniaxial behavior of asphalt concrete. Mech Mater 24:241-255

26. Keenan KE, Pal S, Lindsey DP, Besier TF, Beaupre GS (2013) A viscoelastic constitutive model can accurately represent entire creep indentation tests of human patella cartilage. J Appl Biomech 29:292-302
27. Simo JC (1987) On a fully three-dimensional finite-strain viscoelastic damage model: formulation and computational aspects. Comput Methods Appl Mech Eng 60:153-173

28. Ward IM, Hadley DW (1993) An introduction to the mechanical properties of solid polymers

29. De Crevoisier J, Besnard G, Merckel Y, Zhang H, Vion-Loisel F, Caillard J, Berghezan D, Creton C, Diani J, Brieu M et al (2012) Volume changes in a filled elastomer studied via digital image correlation. Polym Test 31:663-670

30. Kraus G, Childers C, Gruver J (1967) Properties of random and block copolymers of butadiene and styrene. I. Dynamic properties and glassy transition temperatures. J Appl Polymer Sci 11:15811591

31. Diaz R, Diani J, Gilormini P (2014) Physical interpretation of the Mullins softening in a carbon-black filled SBR. Polymer 55:4942-4947

32. Gray GIII, Blumenthal WR (2000) Split-Hopkinson pressure bar testing of soft materials. ASM Handbook 8:488-496

33. Siviour CR, Walley SM (2018) Inertial and frictional effects in dynamic compression testing. In: The Kolsky-Hopkinson bar machine, pp 205-247. Springer

34. Gorham D (1989) Specimen inertia in high strain-rate compression. J Phys D Appl Phys 22:1888

35. Kapoor R, Nemat-Nasser S (1998) Determination of temperature rise during high strain rate deformation. Mech Mater 27:1-12

Publisher's Note Springer Nature remains neutral with regard to jurisdictional claims in published maps and institutional affiliations. 